\title{
The Influence of Glutamate on Axonal Compound Action Potential In Vitro
}

\author{
Ahmed Abouelela ${ }^{1,2}$ Andrzej Wieraszko $1,2,3$ \\ ${ }^{1}$ Department of Biology, The College of Staten Island, City University \\ of New York, New York, NY, USA \\ 2 Program in Neuroscience, The College of Staten Island, \\ City University of New York, New York, NY, USA \\ ${ }^{3}$ Graduate Center, City University of New York, New York, USA \\ Address for correspondence Andrzej Wieraszko, PhD, Department of \\ Biology, Program in Neuroscience, The College of Staten Island, \\ City University of New York, New York, NY 10314, USA \\ (e-mail: Andrzej.Wieraszko@csi.cuny.edu).
}

J Brachial Plex Peripher Nerve Inj 2016;11:e29-e37.

\begin{abstract}
Background Our previous experiments demonstrated modulation of the amplitude of the axonal compound action potential (CAP) by electrical stimulation. To verify assumption that glutamate released from axons could be involved in this phenomenon, the modification of the axonal CAP induced by glutamate was investigated.

Objectives The major objective of this research is to verify the hypothesis that axonal activity would trigger the release of glutamate, which in turn would interact with specific axonal receptors modifying the amplitude of the action potential.

Methods Segments of the sciatic nerve were exposed to exogenous glutamate in vitro, and CAP was recorded before and after glutamate application. In some experiments, the release of radioactive glutamate analog from the sciatic nerve exposed to exogenous glutamate was also evaluated.

Results The glutamate-induced increase in CAP was blocked by different glutamate receptor antagonists. The effect of glutamate was not observed in Ca-free medium, and was blocked by antagonists of calcium channels. Exogenous glutamate, applied to the segments of sciatic nerve, induced the release of radioactive glutamate analog, demonstrating glutamate-induced glutamate release. Immunohistochemical examination revealed that axolemma contains components necessary for glutamatergic

\section{Keywords}

- compound action potential

- glutamate

- axonal plasticity

\section{neurotransmission.}

Conclusion The proteins of the axonal membrane can under the influence of electrical stimulation or exogenous glutamate change membrane permeability and ionic conductance, leading to a change in the amplitude of CAP. We suggest that increased axonal activity leads to the release of glutamate that results in changes in the amplitude of CAPs.
\end{abstract}

\section{Introduction}

Information transfer in the nervous system occurs through generation of action potentials in neurons and synaptic potentials at synaptic junctions. It is known that the magnitude of the potentials generated at the synapses can be modified by their prior activity expressing phenomenon of synaptic plasticity. The best-known examples of synaptic plasticity considered to be the basis of learning are longterm potentiation (LTP) $)^{1,2}$ and long-term depression (LTD). ${ }^{3}$

While postsynaptic activation contributes to the generation of evoked potentials, it has to be preceded by the action potential generated at the axon hillock. Although the action potential has been always considered as a steady signal, resistant to any stimulation-dependent modifications, some recently published results, ${ }^{4-6}$ including the data from our received

July 13, 2016

accepted

August 14, 2016
Dol http://dx.doi.org/

10.1055/s-0036-1593441. ISSN 1749-7221. (c) 2016 Georg Thieme Verlag KG
Stuttgart . New York

License terms

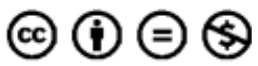


laboratory, ${ }^{7}$ indicate that the properties of the action potential may be modified by axonal activity induced by either electrical or magnetic stimulation.

According to the classical definition, a neurotransmitter is a molecule which is released from presynaptic nerve terminals in response to stimulation and subsequently binds to postsynaptic or presynaptic receptors. However, this classical view has been challenged by several reports, ${ }^{5,6}$ indicating that axons can also release neurotransmitter-like substances in a $\mathrm{Ca}^{2+}$-dependent way without involvement of the synapses. $^{8-11}$ Exposure of sciatic nerve segments to a specific pattern of magnetic ${ }^{7,12}$ or electrical ${ }^{13}$ stimulation increased the amplitude of the evoked compound action potentials (CAPs) and simultaneously enhanced the release of glutamate. ${ }^{7}$ As these nerve preparations contained neither synaptic connections nor cell bodies, the mechanism of the observed amplification had to be intrinsic to the axon.

\section{Materials and Methods}

\section{Animals}

CD-1 mice of both sexes, 1 to 3 months old, kept in normal day light/dark cycle were used in all experiments. All procedures and number of the animals were approved by the Institutional Animal Care and Use Committee of the College of Staten Island/CUNY, and were in conformity with National Institutes of Health Guidelines

\section{Preparation of Sciatic Nerve}

The animals were sacrificed by cervical dislocation, both hind limbs were skinned, and sciatic nerves were dissected out. The nerves were cut into 7 to $10 \mathrm{~mm}$ segments and placed in oxygenated (5:95\%; $\left.\mathrm{CO}_{2} / \mathrm{O}_{2}\right)$ Ringer's solution $\left(33^{\circ} \mathrm{C}\right) \mathrm{com}-$ posed of (in mM): $\mathrm{NaCl} 124, \mathrm{KCl} 3.1, \mathrm{KH}_{2} \mathrm{PO}_{4} 1.3, \mathrm{MgSO}_{4} 1.3$, $\mathrm{CaCl}_{2}$ 3.1, $\mathrm{NaHCO}_{3} 25.5$, and glucose 10 . In some experiments, Ringer's solutions with reduced magnesium concentration (0.32 $\mathrm{mM} \mathrm{MgSO}_{4}$ ) or calcium-free Ringer's solutions were used.

\section{Electrophysiological Recordings}

The extracellular CAP was recorded with iridium microelectrode $(0.180-\mathrm{mm}$ shaft diameter; $1-2-\mu \mathrm{m}$ tip; $5.0 \mathrm{M} \Omega$; WPI, Sarasota, Florida, United States) placed inside the epineurium at the end of the segment.

A stainless steel bipolar stimulating electrode $(500-\mu \mathrm{m}$ shaft diameter; $100-\mu \mathrm{m}$ tip; FHC, Maine, United States) was localized outside of epineurium. The distance between the stimulating and recording electrodes varied in different experiments (5-7 $\mathrm{mm}$ ) and was adjusted to obtain good quality recording, with CAP clearly separated from the stimulus artifact. The nerves were stimulated in all experiments with the frequency of $0.03 \mathrm{~Hz}$. LTP program ${ }^{14}$ was used to generate the pulses which were applied during entire experiment. Different chemicals were added to the recording chamber following stabilization of the potential. The record of extracellular activity was passed through a standard head stage, amplified (Neuro Amp EX, ADInstruments, Inc, Colorado, United States) and stored for further analysis.

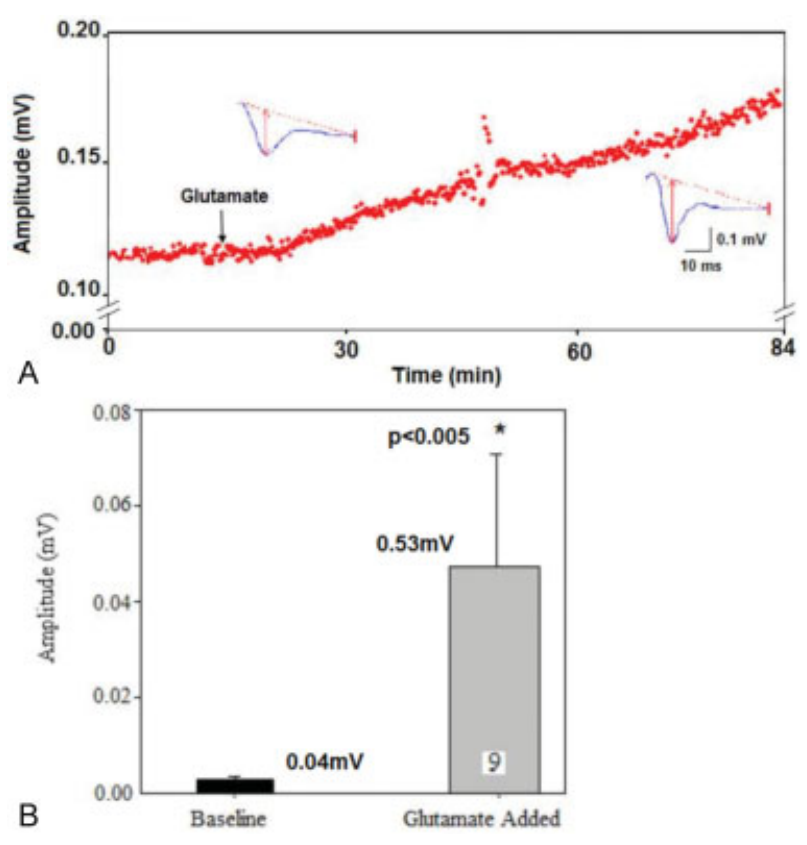

Fig. 1 Glutamate-induced amplification of the amplitude of CAP. (A) An individual experiment demonstrating the effect of addition of 100$\mu \mathrm{M}$ glutamate. The glutamate was added to the recording chamber following 10-minute period of baseline stabilization. Red broken lines are imaginary and were generated by the computer to calculate the amplitude and the area and CAP (see the Materials and Methods section for details). (B) Averaged results of nine experiments testing the influence of exogenous glutamate on CAP. Each bar represents mean \pm SEM, before and after addition of glutamate, respectively. The number of experiments $(n)$ in this and all subsequent figure legends is shown inside of each bar.

\section{The Latency and the Area Measurements}

The latency was measured as the time elapsing between the start of the stimulus and the beginning of the first negativity of the CAP. To measure the area, an imaginary line was drawn by the computer between the first positivity of the potential and the first point of the stable baseline after recording of CAP. This first point of baseline stability was automatically determined by the computer usually after recording of three to four CAPs ( - Fig. 1A). Then the area contained by this imaginary line and the shape of the potential were measured on the hard copy of the recordings with planimeter, expressed in $\mathrm{mm}^{2}$ and taken for further analysis (-Fig. 1A). Four recordings of each parameter of CAP were averaged before and after the treatment.

\section{Measurements of Glutamate-Induced ${ }^{3} \mathrm{H}-\mathrm{D}$-Aspartate Release}

The sciatic nerve ( $\sim 2 \mathrm{~cm}$ long) have been dissected out, and placed in the perfusion chamber filled with constantly oxygenated Ringer's solution. The perfusion chamber was connected to Brandel Suprafusion system. Following 3-hour preincubation in Ringer's containing $3 \mu \mathrm{Ci}$ of ${ }^{3} \mathrm{H}$-D-Asp (Radioactive Chemicals, American Radiolabeled Chemicals, Inc. St. Louis, Missouri, USA), the perfusion of the sciatic nerve was initiated with simultaneous collection of 2-minute fractions into scintillation vials. Separate experiments revealed that the sciatic nerve preincubated in Brandel's chamber for 4 
hours continues to express evoked activity. At vial number 8 , the exogenous glutamate has been injected into perfusion system. The amount of D-Asp was evaluated in all vials in the scintillation counter. The increase in the release of ${ }^{3} \mathrm{H}-\mathrm{D}-\mathrm{Asp}$ was expressed as the area under the peak of release divided by total area under the curve representing the release.

\section{Immunohistochemistry}

The nerves were fixed in $4 \%$ paraformaldehyde for 24 hours and subsequently placed in $30 \%$ sucrose solution for 24 hours. Then, 30- $\mu \mathrm{m}$ cryosections were prepared and transferred onto slides and washed with phosphate-buffered saline (0.01 M, pH 7.4) three times (10 minutes each), permeabilized, and blocked with $2 \%$ nonfat dry milk in phosphate buffer saline, containing $0.02 \%$ TritonX-100 and $10 \%$ normal goat serum for 1 hour. Next, the sections were incubated overnight (at $4^{\circ} \mathrm{C}$ ) with the primary antibody after three washes with phosphate buffer saline. The in vitro expression of various channels and receptors was analyzed with the following antibodies (diluted in nonfat dry milk-phosphate buffer saline with $2 \%$ normal goat serum): rabbit anti-GluR 2/ 3 (1:500; Millipore Corp., Massachusetts, United States), mouse anti-EAAC1 (1:500; Chemicon International Inc., California, United States), mouse anti-nonfat dry milk R2B (1:500), rabbit anti-Ca (1:500), mouse anti-Na (1:500), and rabbit anti-AMPA (1:500). The following day, the samples were blocked for 1 hour with $10 \%$ normal goat serum in nonfat dry milk-phosphate buffer saline, and then washed and incubated for 1 hour at room temperature with secondary antibodies diluted in nonfat dry milk-phosphate buffer saline, with $2 \%$ normal goat serum. The dilution of the secondary antibodies were 1:250 Cy5 (Jackson ImmunoResearch laboratories, Inc., Pennsylvania, United States) (goat anti-mouse) and Alexa fluor 488 (1:250; Santa Cruz Biotechnology, Inc., California, United States) (goat anti-rabbit). The nuclei were counterstained and finally cover-slipped with Prolong Gold antifade reagent with 4',6-diamidine-2-' phenylindole dihydrochloride (DAPI, Invitrogen, Eugene, Oregon, United States). Images were captured using confocal microscopy (Leica SP2 AOBS) using a 488-nm Ar/Kr laser for the Alexa fluor 488,543-nm HeNe laser for Cy5 and 405-nm diode laser for DAPI. Images were reconstructed from $\mathrm{Z}$ stack using Leica software.

\section{The Evaluation of Intracellular Calcium with Imaging Quantitative Measurements}

Fluo3 $(5 \mu \mathrm{M})$ was added to the nerve-containing dish for 30 minutes. To determine baseline $\left[\mathrm{Ca}^{2+}\right]$, three frames before the addition of the drugs were taken and the fluorescence intensity of these images was used as baseline. The changes in the fluorescent intensity in subsequent images after addition of the glutamate were expressed as percentage of the baseline. Confocal images of cellular fluorescence were obtained using a Leica inverted microscope (Leica SP2 AOBS) equipped with a dry $10 \times$ HC PL fluotar $10 \times 0.3$ dry objectives. The excitation wavelength used was 496-nm $\mathrm{Ar} / \mathrm{Kr}$ laser, and the emission wavelength of the ionophore for the intensity measurement was 509 to $596 \mathrm{~nm}$. All recordings were performed at room temperature $\left(22-25^{\circ} \mathrm{C}\right)$. Three base scans were recorded and then recording chamber was loaded with one of the following: glutamate $(100 \mu \mathrm{M}), N$-methyl-D-aspartate (NMDA) $(10 \mu \mathrm{M})$, or MK801 $(50 \mu \mathrm{M})$, and the images were then taken every 30 seconds. The changes in free calcium concentration inside the axon were evaluated by comparison of the baseline fluorescent intensity with the intensity after addition of given molecule. Data were expressed as percent changes in fluorescent intensities.

\section{Chemicals Used}

Chemicals purchased from Sigma:NMDA; $\alpha$-amino-3-hydroxy-5-methyl-4-isoxazolepropionic; kainic acid; kynurenic acid; dizocilpine; 6-cyano-7-nitroquinoxaline-2, 3-dione. All other standard molecules are used to prepare standard solutions. Chemicals purchased from Tocris: DL-threo- $\beta$-benzyloxyaspartate; verapamil; sodium dantrolene. Chemical from Invitrogen: Fluo-3.

\section{Statistics}

All data were postanalyzed offline and statistics were computed in Statistica V 6.1 (Statsoft, Inc. Tulsa, Oklahoma, United States) and MatLab software. Data were analyzed with analysis of variance (ANOVA) and paired $t$-test unless specified otherwise. All data are expressed as mean \pm SEM.

\section{Results}

\section{The Modification of CAP Characteristics by Exogenous Glutamate}

Following stabilization of CAP amplitude, glutamate $(100 \mu \mathrm{M})$ was added to the recording chamber. As depicted in - Fig. 1A, CAP amplitude started to increase approximately 10 minutes after addition of glutamate and gradually reached its maximum approximately 75 minutes after addition of glutamate. To standardize experimental conditions, all experiments were terminated 75 minutes after addition of glutamate. The amplitude of 10 potentials recorded at the end of the experiment was averaged and taken as an indicator of amplification. - Fig. 1B illustrates mean CAP amplitude before $(0.04 \pm 0.01 \mathrm{mV})$ and after $(0.53 \pm 0.41 \mathrm{mV})$ addition of glutamate $(p<0.005, n=9$, $t$-test). The increase in the amplitude of CAP was accompanied by decrease in CAP latency. As depicted in - Fig. 2A, the mean latency was reduced from $3.91 \mathrm{~ms}$ recorded before glutamate addition to $3.74 \mathrm{~ms}$ observed at the end of the experiment ( $p<0.00003, n=10, t$-test). In parallel with increased amplitude and decreased latency, the area of CAP was significantly enhanced from $0.670 \mathrm{~cm}^{2}$ before glutamate application to 0.682 $\mathrm{cm}^{2}$ after glutamate addition ( $p<0.002, n=12$, - Fig. 2B).

\section{Inhibition of Glutamate-Induced CAP Amplification}

Kynurenic acid, a general antagonist of glutamergic receptors, blocked glutamate-induced amplification, but it did not influence the amplitude of CAP by itself ( - Fig. 3A, middle bar; CAP $0.08 \pm 0.02 \mathrm{mV}$ both before and after glutamate application, $p=0.7, n=10$ ). - Fig. 3A (right bar) illustrates the averaged results of 10 experiments aimed to evaluate the effects of glutamate in the presence of kynurenic acid (mean 


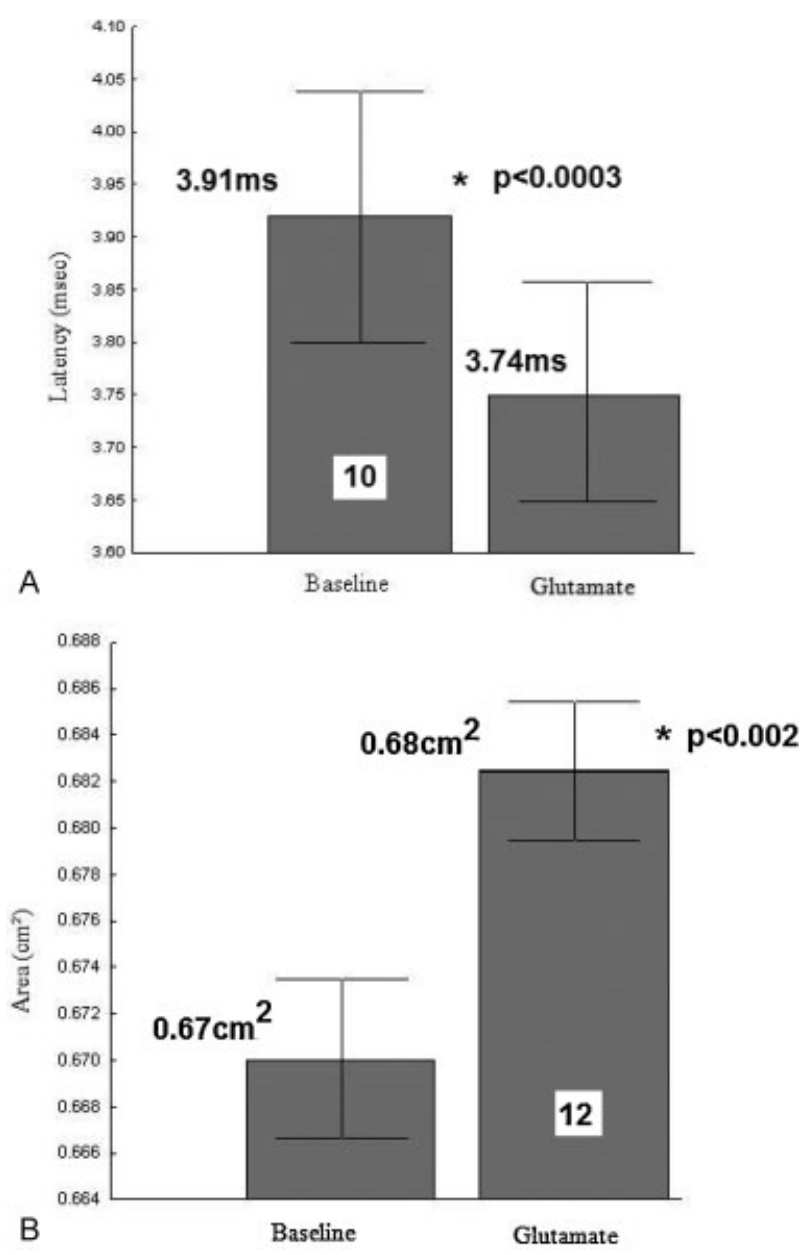

Fig. 2 The influence of glutamate on the latency (A) and area (B) of CAP. (A) Averaged results of 10 experiments illustrating the influence of glutamate on the latency of CAP. (B) Averaged results of 12 experiments, displaying the effect of glutamate on the area of CAP.

before $=0.07 \pm 0.02 \mathrm{mV}$, mean after $=0.06 \pm 0.02 \mathrm{mV}$; $p<0.04, n=10$ ). Thus, blocking glutamate receptors does not affect baseline axonal transmission.

Selective antagonist of NMDA receptor, MK801, had no effect on CAP amplitude, but eliminated the effect of glutamate added later ( $\boldsymbol{- F i g}$. $\mathbf{3 B}$, the middle and right bars, $p=0.515, n=5$ ). Similarly, CNQX (an antagonist of AMPA/ kainate receptors) prevented the effects of glutamate. As depicted in - Fig. 3 C, there is no statistical difference between the averaged amplitudes of CAP recorded in the presence of CNQX alone and in the presence of glutamate $(p=0.745$, $n=7$ ). A slight attenuation of CAP following addition of CNQX was not statistically significant.

\section{The Influence of Glutamate Receptor Agonists on the Amplitude of CAP}

The mixture of $10-\mu \mathrm{M}$ NMDA and $10-\mu \mathrm{M}$ kainate (agonists of glutamergic receptors) added to the recording chamber significantly increased CAP amplitude (-Fig. 4). The mean values of CAP amplitude before and after the addition of NMDA and kainate were $0.19 \pm 0.059$ and
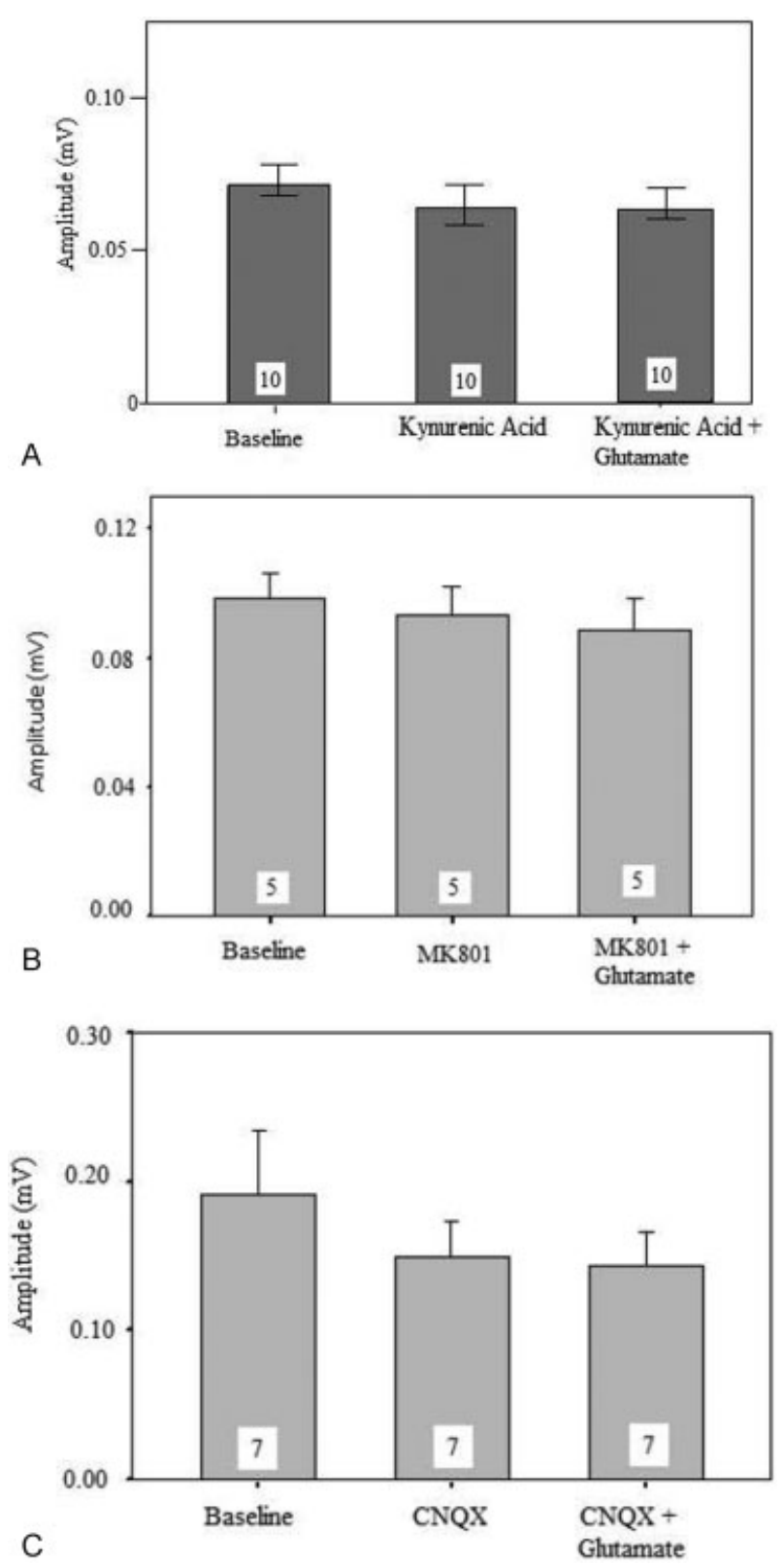

Fig. 3 Blocking the effects of glutamate by glutamate receptors antagonists. (A) The average results of 10 experiments demonstrating the elimination of glutamate effect on CAP in the presence of kynurenic acid (2 mM). (B) Averaged results of five experiments demonstrating the elimination of glutamate effect by MK801 $(50 \mu \mathrm{M})$. (C) Averaged results of seven experiments showing the elimination of the effects of glutamate by CNQX (250 $\mu \mathrm{M})$. In each of the figure, the left bar shows the control potential (baseline), the middle bar illustrates the averaged amplitude of CAP 25 minutes after addition of tested antagonist itself, and the right bar shows the amplitude of CAP 25 minutes after addition of glutamate in the presence of antagonist. Every antagonist was added to the incubation chamber 10 minutes prior addition of glutamate.

$0.258 \pm 0.0662 \mathrm{mV}$, respectively $(p<0.005, n=8)$. These data support the view that glutamate mediates its actions on the CAP through activation of the AMPA/kainate and NMDA receptors. 


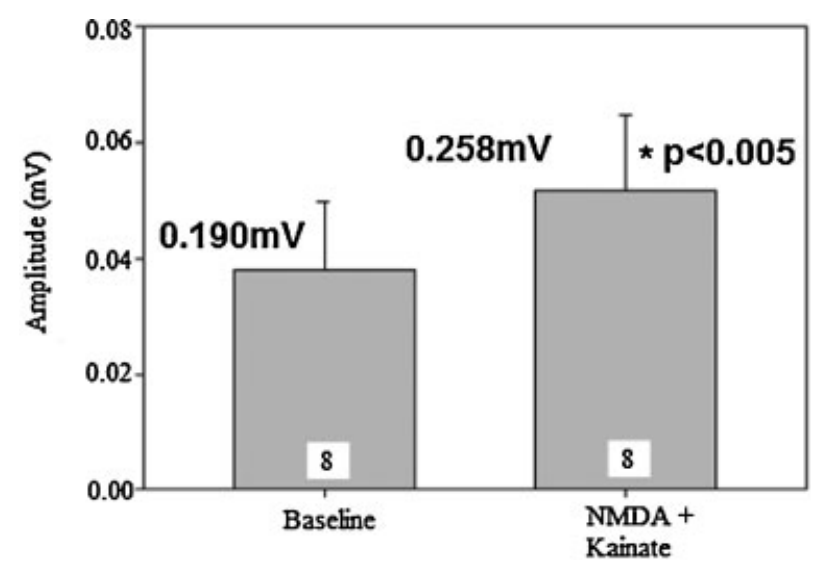

Fig. 4 The combined effect of glutamate receptor agonists, NMDA and kainate. Averaged results of eight experiments showing the effect

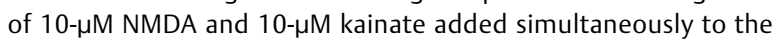
segments of sciatic nerve incubated in reduced $\mathrm{Mg}$ environment.

\section{The Role of Calcium in Glutamate-Induced CAP Increase} The addition of glutamate in $\mathrm{Ca}^{2+}$-free Ringer's solution had no effect on CAP amplitude (-Fig. 5A). The mean values of CAP recorded before and after addition of glutamate were $0.083 \pm 0.016$ and $0.072 \pm .0122 \mathrm{mV}$, respectively
( $p=0.060, n=8$ ). In agreement with the results of these experiments, verapamil prevented glutamate-induced increase in CAP ( $\mathbf{- F i g}$. 5B; $p=0.529, n=6$ ). Since glutamate applied in the presence of dantrolene also had no influence on the amplitude of CAP ( $p=0.623, n=6$, - Fig. $5 \mathbf{C}$ ), one can assume that the calcium released from endoplasmic reticulum may additionally participate in the action of exogenous glutamate. As in the case of verapamil, dantrolene itself had no effect on CAP (-Fig. $\mathbf{5 C}$, middle bar). Moreover, the experiments with calcium channel antibody revealed that there are indeed voltage-sensitive calcium channels present on the surface of the sciatic nerve (-Fig. 5D).

\section{Glutamate-Induced Release of Radioactive Glutamate Analog (2,3- ${ }^{3} \mathrm{H}$-D-Aspartate) from the Segments of Sciatic Nerve In Vitro}

If exogenous glutamate exerts influence on the amplitude of the action potential, similar action could be expressed by endogenous glutamate released from axons during intense activity. This action of endogenous glutamate could be further amplified by the glutamate-induced glutamate release. To verify this assumption, we tested the effect of exogenous glutamate on the release of radioactive analog of glutamate ${ }^{3} \mathrm{H}-\mathrm{D}$-Asp from the segments of the sciatic nerve. As depicted

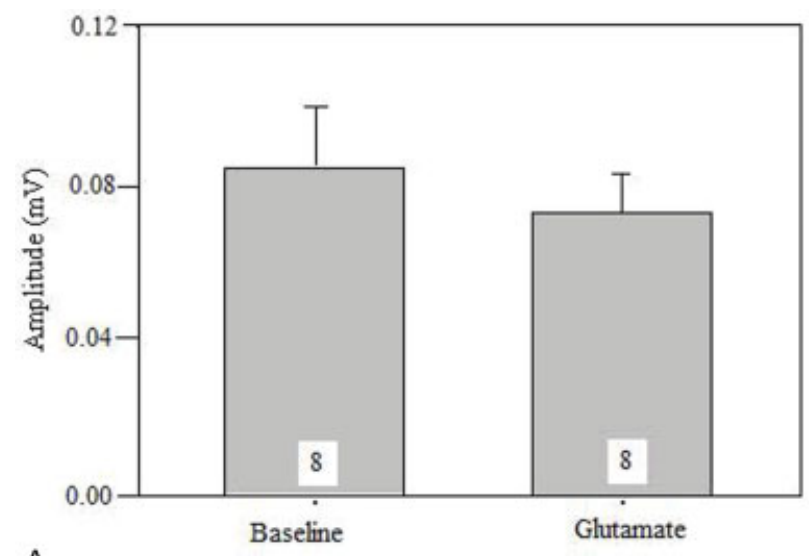

A

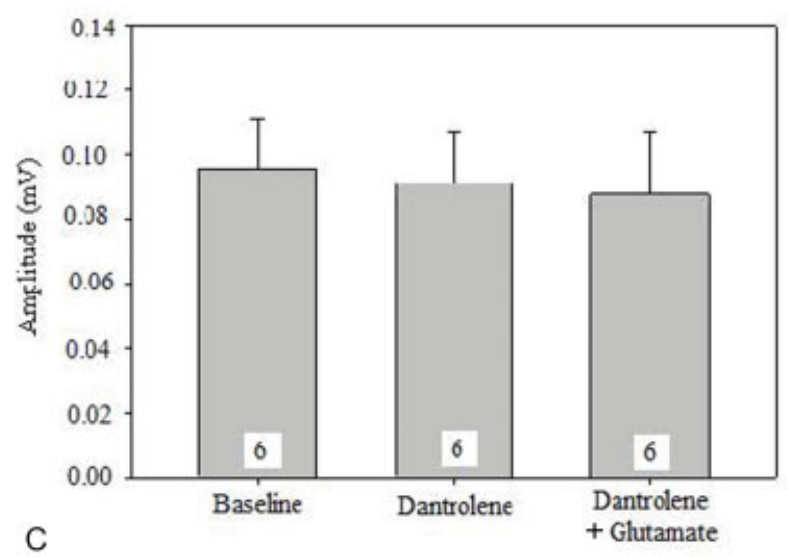

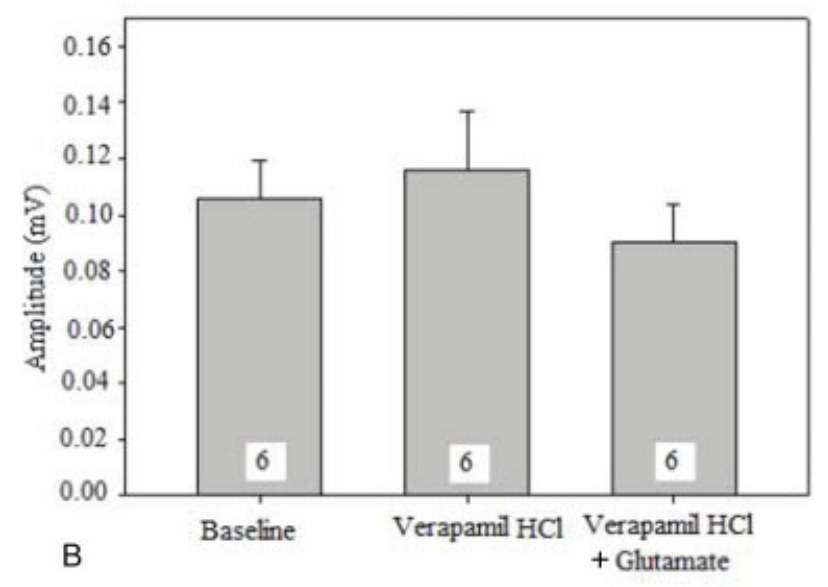

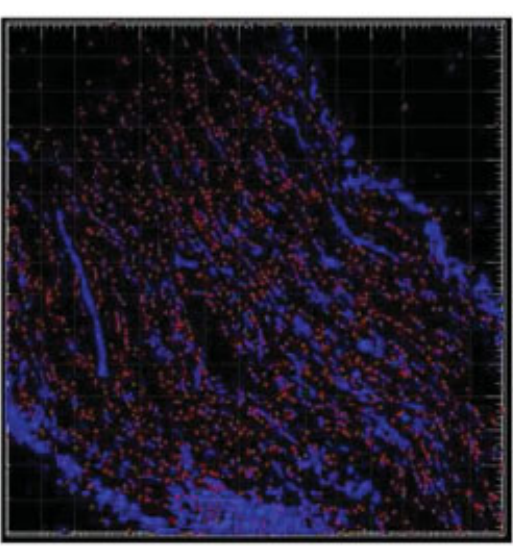

Fig. 5 The involvement of calcium ions in the glutamate-induced effects. (A) Averaged results of eight experiments showing no effect of glutamate in $\mathrm{Ca}^{2+}$-free environment. (B) The blocker of voltage-gated calcium channels verapamil $(10 \mu \mathrm{M})$ and the inhibitor of intracellular calcium channels dantrolene $(10 \mu \mathrm{M})(\mathrm{C})$ prevented the enhancement of CAP by subsequent addition of $100-\mu \mathrm{M}$ glutamate. (D) Expression of voltage-gated calcium channels on the surface of axolemma. The section of sciatic nerve of 1- to 3-month-old CD-1 mice immunostained with antibody against calcium channels (red). 

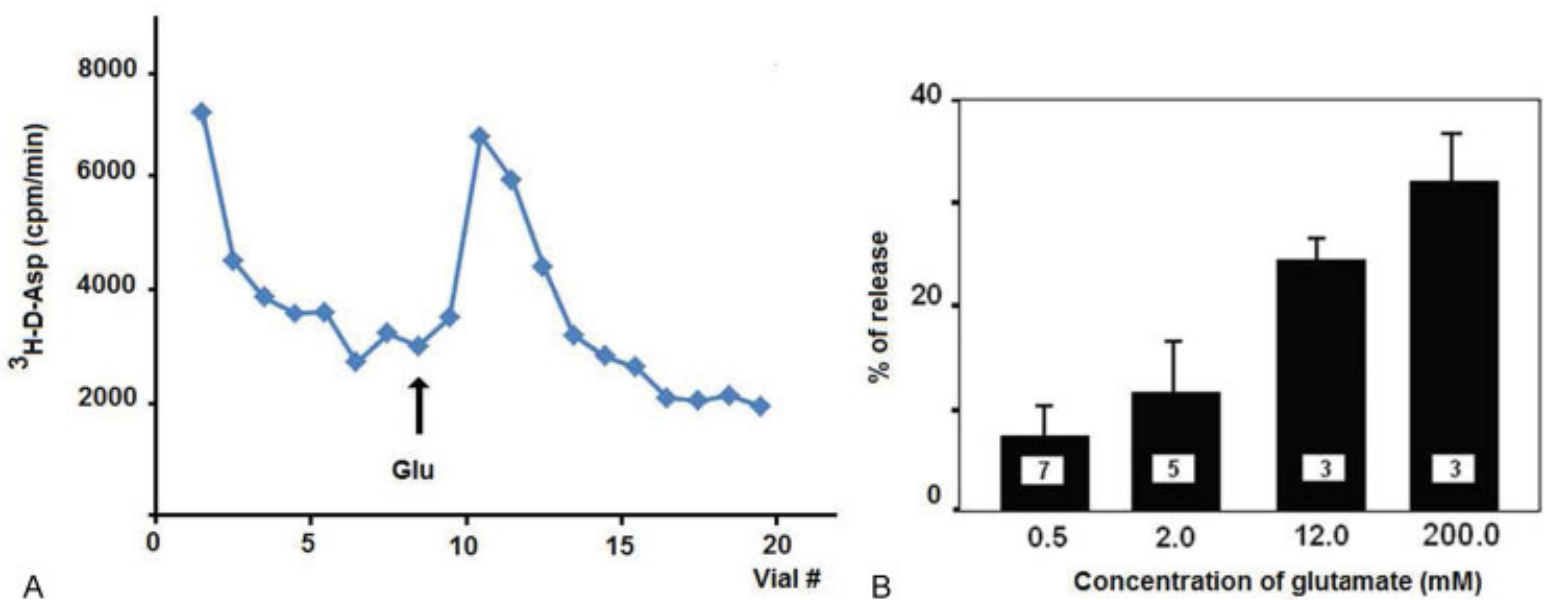

Fig. 6 Glutamate-induced ${ }^{3} \mathrm{H}$-D-aspartate release. (A) An example of individual experiment. Following preincubation for 3 hours with $2,3{ }^{3} \mathrm{H}-\mathrm{D}$-aspartate, the segments of sciatic nerve have been perfused with Ringer's solution continuously for the rest of the experiment. The perfusate was collected in individual vials of the fraction collector ( 2 minutes for each vial). Then at vial \# 8 , the glutamate $(2.0 \mathrm{mM})$ has been injected into perfusion Ringer's. The radioactivity in each vial has been determined at the end of the experiment with scintillation counter. (B) Averaged results of the release experiments illustrating the relation between the magnitude of the release and glutamate concentration.
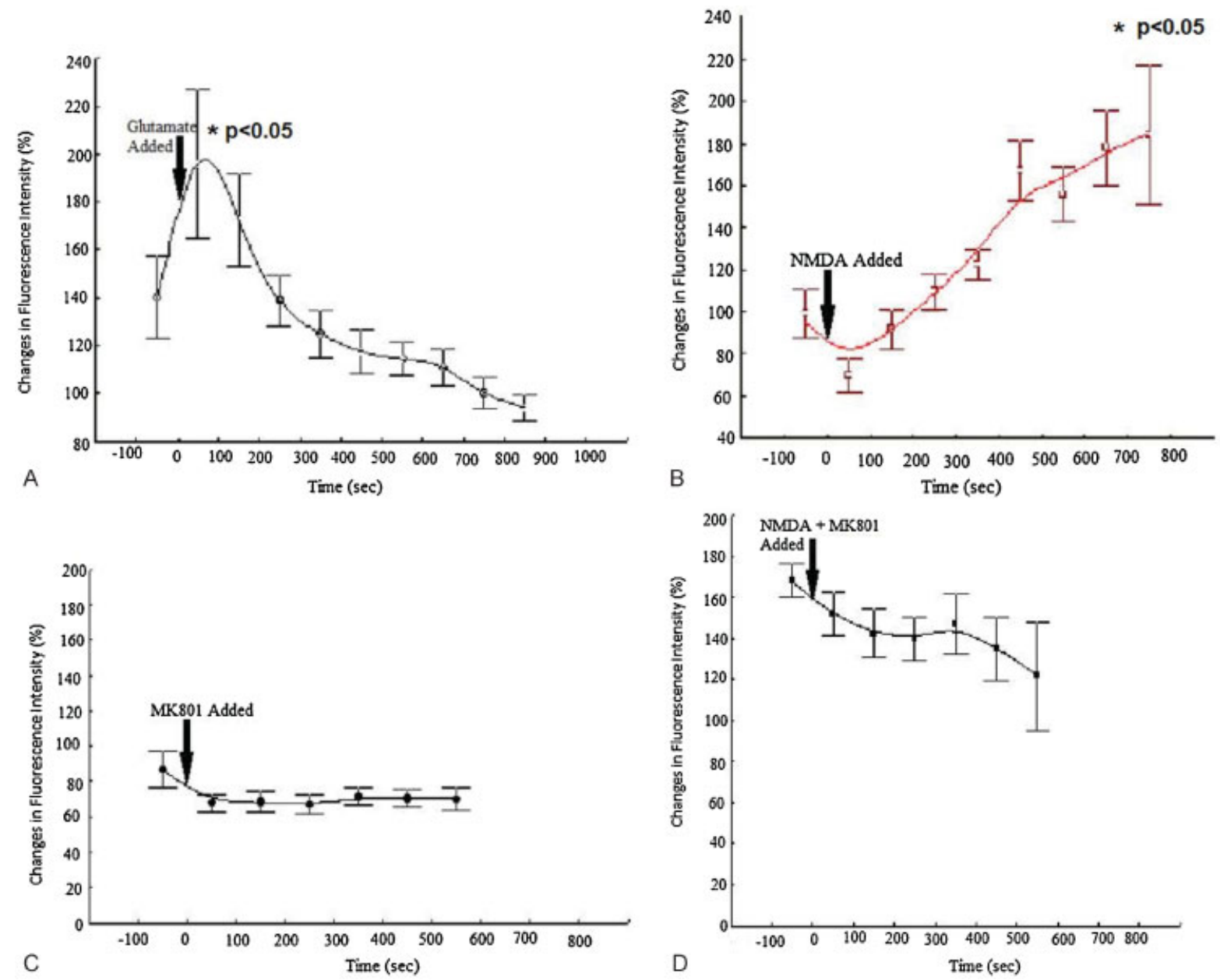

Fig. 7 The effect of activation of glutamate receptors on the intracellular level of free calcium. (A) Addition of glutamate (100 $\mu \mathrm{M})$ temporarily enhanced the intracellular concentration of free calcium. The level of calcium increased during the first 30 seconds after addition of glutamate to decline rapidly shortly after. This transient increase was statistically significant $(n=3)$. (B) The influence of NMDA $(100 \mu \mathrm{M})$. There was significant increase in fluorescence intensity after the addition of NMDA $(n=3)$. The rise continued till the end of the experiment. (C) While MK801 added by itself had no effect on the intracellular calcium level, MK801 added together with NMDA (D) showed a slight tendency to attenuate the intracellular calcium concentration $(n=3$, ns). 
in - Fig. 6A, the application of exogenous glutamate induced the release of ${ }^{3} \mathrm{H}$-D-aspartate. The increase in the release was transient and the amount of radioactivity quickly came back to the baseline level observed before glutamate application. - Fig. 6B illustrates a linear raise in the magnitude of ${ }^{3} \mathrm{H}$-D-aspartate release directly related to an increase in the concentration of applied glutamate.

\section{Quantitative Measurements of Intracellular Calcium Using Calcium Imaging}

As depicted in - Fig. 7A, there was a significant increase in fluorescence intensity within 60 seconds following addition of glutamate ( $p<0.05, n=3$ ). The increase was transient, indicating the presence of buffering mechanism that quickly reduces the concentration of free axoplasmic calcium.

Addition of NMDA, which activates channels permeable for both calcium and sodium, induced slowly developing increase in fluorescence which remained elevated for the rest of the experiment ( $p<0.05, n=3$, - Fig. 7B).
As expected, MK801 completely blocked the NMDA-induced increase in axoplasmic free calcium. There was no increase in the fluorescence intensity neither after the addition of MK801 itself (-Fig. 7C, averaged results of three experiments) nor after the addition of NMDA and MK801 to the recording chamber (-Fig. 7D, averaged results of three experiments).

\section{Immunohistochemical Characterization of Channel Expression on the Axolemma}

The data presented thus far use pharmacological agents to provide functional evidence for the presence of various channels on the membrane of axons within the sciatic nerve. Therefore, we sought to use immunofluorescence to probe for the presence of channels and receptors to correlate morphological, pharmacological, and electrophysiological observations. As anticipated, the axons within the sciatic nerve express high level of voltage-sensitive sodium channels

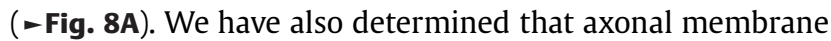
contains all the molecular components necessary for

A
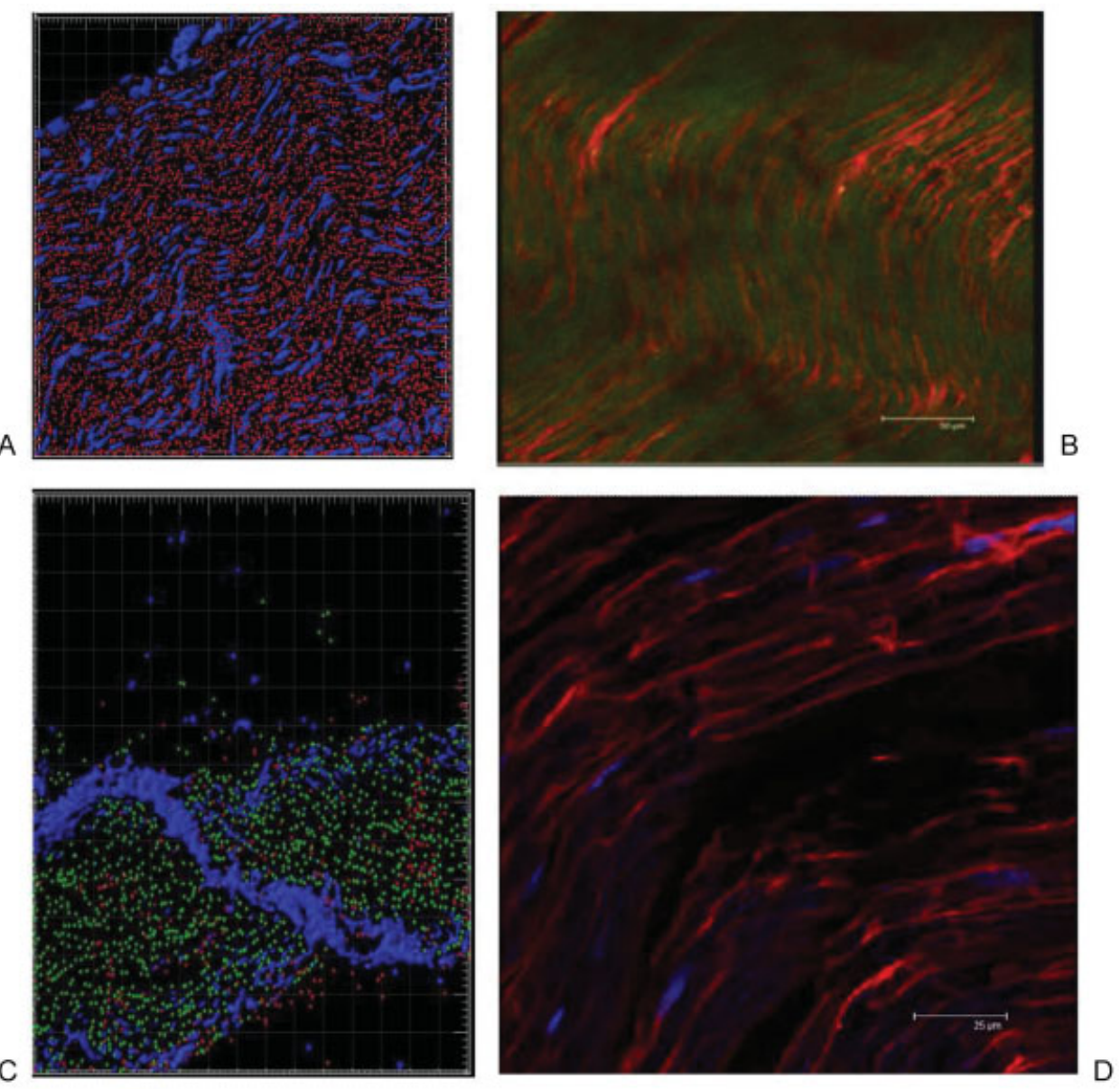

Fig. 8 The axonal expression of molecules essential for generation of the action potential and glutamatergic neurotransmission. (A) The sciatic nerve expresses high level of voltage-sensitive sodium channels (red); (B) The sciatic nerve expresses AMPA/kainate receptors (green) and excitatory amino acid transporter (red); (C) Sections from sciatic nerve immunostained with antibody against NMDA receptors (green) and $\mathrm{mGluR}$ (red); (D) the sections of sciatic nerve expressing NMDA receptor (red). All these images were obtained from sections from sciatic nerve of 1- to 3month-old CD-1 mice immunostained with antibody against specific molecules. 
glutamatergic neurotransmission. - Fig. 8 illustrates the distribution of immunoreactive particles for AMPA/kainate (-Fig. 8B), NMDA (-Fig. 8C, D), and mGlur2 (-Fig. 8C) receptors. We have also found ample immunoreactivity indicating the presence of amino acid transporters (-Fig. 8D).

\section{Discussion}

Although action potentials in peripheral nerves have been always considered as steady signals, resistant to any stimulation-dependent modifications, some recently published results, ${ }^{4-6}$ including the data from our laboratory, ${ }^{7,13}$ indicate that the properties of the action potential may be modified by specific axonal activity. Moreover, it has been shown that sciatic nerve releases glutamate in response to repeated stimulation ${ }^{7}$ and to exogenous glutamate. The results presented here strongly suggest that glutamate released during intense axonal activity would be responsible for the observed CAP amplification. In the currently described experiments, the exogenous glutamate would mimic the action of electrical stimulation.

The addition of exogenous glutamate increased the amplitude of CAPs in sciatic nerve preparations. The glutamateinduced increase of the CAPs was calcium-dependent and could be abolished by glutamate receptors antagonists. Moreover, the segments of sciatic nerve preloaded with radioactive glutamate analog, D-Asp, released this analog in response to exposure to exogenous glutamate. The ability of the segments of axons in vitro to generate evoked action potentials validates our data and makes processes induced by exogenous glutamate very likely to occur in vivo. Immunohistochemical analysis revealed that axolemma of the peripheral nerves is equipped with several proteins important for neuronal communication mediated by glutamate. Apparently, these proteins can under the influence of electrical stimulation or exogenous glutamate change membrane permeability, leading to the changes in the amplitude of the CAPs. These changes demonstrate the existence of axonal plasticity expressed as an alteration in the amplitude of the action potential following periods of variable activity accompanied by axonal release of glutamate. ${ }^{7}$ This glutamate, through paracrine action, can in turn affect adjacent axons, inducing long-lasting modifications in the properties of the nerve. The axonal plasticity would constitute exclusive property of the axon and could contribute together with different forms of synaptic plasticity ${ }^{1,3}$ to use-dependent modifications of the activity of neuronal networks. It is not known exactly what would be extracellular concentration of glutamate during repetitive axonal stimulation, although it is known that it can reach higher micromolar levels during synaptic activity in the central nervous system. ${ }^{15}$ We have selected $100-\mu \mathrm{M}$ concentration of exogenous glutamate for electrophysiological experiments considering the fact that this exogenous glutamate would have to penetrate through epineurium, perineurium, and endoneurium to reach glutamate receptors located on the neuronal and glial cells membranes. ${ }^{16}$ It has to be emphasized that exogenous glutamate injected to the perfusion system was quickly diluted approximately 20fold before it reached the preparation of the sciatic nerve in the perfusion chamber. Therefore, - Fig. 6B shows the effective concentrations of exogenous glutamate in the vicinity of sciatic nerve within the range of 25 to $1,000 \mu \mathrm{M}$.

The transient increase in the extracellular glutamate concentration, occurring due to either repetitive axonal activity or application of exogenous glutamate, would result in temporary elevation of intra-axonal free calcium concentration originating from extracellular and intracellular sources following the opening of different channels. The action of these calcium channels has to be synchronized to induce amplification of CAP. Therefore, one can assume that the intra-axonal increase in calcium concentration has to be compartmentalized. It would correspond to individual calcium channels localized in the proximity of other signaling molecules (e.g., glutamate receptors, $\mathrm{Ca}^{2+}$ channels, nitric oxide synthase), organized along the internodal axolemma under the myelin sheath in discrete "axonal nanocomplexes." Although overactivation of nanocomplexes during disease can lead to a lethal release of $\mathrm{Ca}^{2+}$ from intra-axonal stores, ${ }^{17,18}$ the discreet interaction of exogenous glutamate with these nanocomplexes and subsequent controlled elevation of intracellular calcium concentration could explain the increase in CAP in our experiments. The role of NMDA receptors, which seem to be crucial for axonal plasticity, supports our assumption. The addition of glutamate or NMDA in regular Ringer's did not induce an increase in intracellular calcium level unless the concentration of magnesium has been reduced, facilitating opening of NMDA receptor-linked channel. Moreover, while an increase in intracellular calcium concentration lasted $60 \mathrm{sec}$ onds only after addition of glutamate, calcium stayed elevated for approximately 900 seconds following NMDA addition. Thus, it is likely that selective activation of NMDA receptors by NMDA activates ionotropic mechanisms and only then an increase in CAP is induced. The discrepancy between duration of glutamate and NMDA effects can be also related to the various channels activated by the addition of glutamate. While NMDA selectively activates only the NMDA receptors, glutamate activates both ionotropic and metabotropic receptors. ${ }^{19}$ As some of the later are inhibitory, simultaneous activation of different types of glutamate receptors can yield attenuation of free calcium release.

The leading role of NMDA receptors in axonal plasticity noticed by us was also reported by others. Matsumoto et $\mathrm{al}^{20}$ found that $100-\mu \mathrm{M}$ glutamate and 100- $\mathrm{MM}$ NMDA increased the CAP recorded from dorsal column axons of neonatal rats.

As demonstrated by Nikolaeva et $\mathrm{al}^{21}$ the release of calcium from intra-axonal stores is highly dependent on $\mathrm{Na}^{+}$influx and reversal of the mitochondrial $\mathrm{Na}^{+} / \mathrm{Ca}^{2+}$ exchanger. ${ }^{21}$ Although this observation suggests that both of these pathways contribute to the intra-axonal calcium concentration and work independently, ${ }^{21}$ mitochondrial calcium is not likely to be involved in CAP increase observed in our experiments. Dantrolene, which blocks IP3 receptor-linked intracellular calcium channels, completely eliminated the increase of CAP. Therefore, we would like to suggest that influx of calcium via NMDA receptors-linked channels initiates calcium release from internal, ryanodine receptor-regulated channels by the mechanism described for cardiac muscle. 22

The reduced latency and increased area of CAP after glutamate application could be explained in view of the 
results reported by Sasaki et al. ${ }^{23}$ They have determined that individual action potentials recorded from hippocampal single axons increased their duration following application of exogenous glutamate. The glutamate-induced axonal plasticity may, as a consequence of axonal repeated activation, represent beneficiary effect exerted in neuromuscular system by extensive physical activity. Indeed, the presence of several ions channels, carriers, and receptors visualized in axonal membranes by us apparently creates environment permissible for modification of the action potential during its propagation down the axonal length.

Our novel data together with published results clearly indicate that in spite of prevailing notion about "all-ornothing" property of the action potential, axons and action potentials are capable of conveying the information in an analog manner. ${ }^{24}$

\section{Conclusion}

Repetitive generation of the action potentials in the peripheral nerve would result in nonsynaptic, glial, and/or axonal release of glutamate. This release could occur at activated nanocomplexes, which would mimic the synaptic action, releasing glutamate. Released glutamate would further enhance its action by glutamate-induced glutamate release. The transient increase in extracellular glutamate would in turn activate calcium-dependent processes, which would result in an increase in the amplitude of CAP. This phenomenon, representing axonal plasticity, could explain beneficial effects of repetitive muscle activation on the recovery from the injury.

\section{Acknowledgments}

We thank Drs Ahmed and El-Idrissi for valuable discussions during the course of this research. This work was supported by PSCCUNY grants 636440041 and 656140043 , and $\mathrm{PhD}$ fellowship from Graduate Center/City University of New York to Ahmed Abouelela.

\section{References}

1 Bliss TV, Collingridge GL. A synaptic model of memory: longterm potentiation in the hippocampus. Nature 1993;361(6407): 31-39

2 Kandel ER. The molecular biology of memory storage: a dialogue between genes and synapses. Science 2001;294(5544): 1030-1038

3 Dudek SM, Bear MF. Homosynaptic long-term depression in area CA1 of hippocampus and effects of $\mathrm{N}$-methyl-D-aspartate receptor blockade. Proc Natl Acad Sci U S A 1992;89(10):4363-4367

4 Carp JS, Chen XY, Sheikh H, Wolpaw JR. Operant conditioning of rat $\mathrm{H}$-reflex affects motoneuron axonal conduction velocity. Exp Brain Res 2001;136(2):269-273
5 Meeks JP, Mennerick S. Selective effects of potassium elevations on glutamate signaling and action potential conduction in hippocampus. J Neurosci 2004;24(1):197-206

6 Debanne D. Information processing in the axon. Nat Rev Neurosci 2004;5(4):304-316

7 Ahmed Z, Wieraszko A. Activity-dependent axonal plasticity: the effects of electrical stimulation on compound action potentials recorded from the mouse nervous system in vitro. The Open Neurosci 2009;3:14-25

8 Fischer MJ, Reeh PW, Sauer SK. Proton-induced calcitonin generelated peptide release from rat sciatic nerve axons, in vitro, involving TRPV1. Eur J Neurosci 2003;18(4):803-810

9 Sauer SK, Reeh PW, Bove GM. Noxious heat-induced CGRP release from rat sciatic nerve axons in vitro. Eur J Neurosci 2001;14(8): 1203-1208

10 Spitzer MJ, Reeh PW, Sauer SK. Mechanisms of potassium- and capsaicin-induced axonal calcitonin gene-related peptide release: involvement of L- and T-type calcium channels and TRPV1 but not sodium channels. Neuroscience 2008;151(3):836-842

11 Kukley M, Capetillo-Zarate E, Dietrich D. Vesicular glutamate release from axons in white matter. Nat Neurosci 2007;10(3): 311-320

12 Ahmed Z, Wieraszko A. The mechanism of magnetic field-induced increase of excitability in hippocampal neurons. Brain Res 2008; 1221:30-40

13 Ahmed Z, Wieraszko A. The influence of pulsed magnetic fields (PMFs) on nonsynaptic potentials recorded from the central and peripheral nervous systems in vitro. Bioelectromagnetics 2009; 30(8):621-630

14 Anderson WW, Collingridge GL. The LTP Program: a data acquisition program for on-line analysis of long-term potentiation and other synaptic events. J Neurosci Methods 2001;108(1):71-83

15 Stys PK, Lipton SA. White matter NMDA receptors: an unexpected new therapeutic target? Trends Pharmacol Sci 2007;28(11): 561-566

16 Matute C, Alberdi E, Domercq M, et al. Excitotoxic damage to white matter. J Anat 2007;210(6):693-702

17 Ouardouz M, Nikolaeva MA, Coderre E, et al. Depolarizationinduced $\mathrm{Ca} 2+$ release in ischemic spinal cord white matter involves L-type $\mathrm{Ca} 2+$ channel activation of ryanodine receptors. Neuron 2003;40(1):53-63

18 Ouardouz M, Malek S, Coderre E, Stys PK. Complex interplay between glutamate receptors and intracellular $\mathrm{Ca} 2+$ stores during ischaemia in rat spinal cord white matter. J Physiol 2006;577 (Pt 1):191-204

19 Ouardouz M, Coderre E, Basak A, et al. Glutamate receptors on myelinated spinal cord axons: I. GluR6 kainate receptors. Ann Neurol 2009;65(2):151-159

20 Matsumoto M, Sasaki T, Nagashima H, Ahn ES, Young W, Kodama $\mathrm{N}$. Effects of N-methyl-d-aspartate, glutamate, and glycine on the dorsal column axons of neonatal rat spinal cord: in vitro study. Neurol Med Chir (Tokyo) 2005;45(2):73-80, discussion 81

21 Nikolaeva MA, Mukherjee B, Stys PK. Na+-dependent sources of intra-axonal $\mathrm{Ca} 2+$ release in rat optic nerve during in vitro chemical ischemia. J Neurosci 2005;25(43):9960-9967

22 Stirling DP, Stys PK. Mechanisms of axonal injury: internodal nanocomplexes and calcium deregulation. Trends Mol Med 2010;16(4):160-170

23 Sasaki T, Matsuki N, Ikegaya Y. Action-potential modulation during axonal conduction. Science 2011;331(6017):599-601

24 Clark B, Häusser M. Neural coding: hybrid analog and digital signalling in axons. Curr Biol 2006;16(15):R585-R588 